ISSN 0103-5150

Fisioter. Mov., Curitiba, v. 26, n. 2, p. página 307-314, abr./jun. 2013

Licenciado sob uma Licença Creative Commons

\title{
Fatores que influenciam a qualidade de vida de cuidadores de paralisados cerebrais
}

\author{
Factors influencing the quality of life of \\ caregivers of cerebral palsy children
}

\section{Kênnea Martins Almeida ${ }^{[a]}$, Breno Morais da Fonseca ${ }^{[b]}$, Amanda Azevedo Gomes ${ }^{[c]}$, Murilo Xavier Oliveira ${ }^{[\mathrm{d}]}$}

[a] Doutoranda em Ciência da Reabilitação pela Universidade Federal de Minas Gerais (UFMG), professora assistente do Departamento de Fisioterapia Universidade Federal dos Vales do Jequitinhonha e Mucuri (UFVJM), Diamantina, MG Brasil, e-mail: kennea.almeida@gmail.com

[b] Graduado em Fisioterapia pela Universidade Federal dos Vales do Jequitinhonha e Mucuri (UFVJM), fisioterapeuta na Clínica Valemed, Diamantina, MG - Brasil, e-mail: breno-morais@hotmail.com

[c] Graduada em Fisioterapia pela Universidade Federal dos Vales do Jequitinhonha e Mucuri (UFVJM), fisioterapeuta na Clínica de Fisioterapia Fisio \& Shanti Ltda., Diamantina, MG - Brasil, e-mail: gomes_mandinha@hotmail.com

[d] Doutor em Engenharia Biomédica pela Universidade Camilo Castelo Branco, professor assistente do Departamento de Fisioterapia da Universidade Federal dos Vales do Jequitinhonha e Mucuri (UFVJM), Diamantina, MG - Brasil, e-mail: muriloxavier@gmail.com

\section{Resumo}

Introdução: Crianças com Paralisia Cerebral (PC) apresentam uma série de deficiências e limitações que geram dependência em relação a seus cuidadores. Objetivo: Avaliar se a qualidade de vida de cuidadores de crianças com PC está relacionada ao comprometimento motor da criança, à classe econômica ou à escolaridade do cuidador. Materiais e métodos: Foram entrevistados 45 cuidadores informais de crianças com PC por meio do questionário WHOQOL-bref e da classificação econômica da Associação Brasileira de Empresas de Pesquisa (Abep). As crianças foram classificadas de acordo com o Gross Motor Function Classification System. Realizaram-se testes de associação e correlação das variáveis com os domínios do WHOQOL-bref, utilizando-se Teste t independente e Coeficiente de Correlação de Spearman. Resultados: Foi encontrada correlação positiva entre o domínio ambiental do WHOQOL-bref e classe econômica $(0,56)$, e escolaridade do 
cuidador $(0,389)$. Houve diferença significante entre os subgrupos de maior e menor classe econômica nos domínios ambiental $(p=0,001)$ e psicológico $(p=0,031)$, e entre os subgrupos de maior e menor nível escolar no domínio ambiental $(p=0,049)$. Conclusão: $A$ qualidade de vida dos cuidadores parece ser influenciada pelo nível socioeconômico e não pelo nível de função motora da criança com PC.

Palavras-chave: Qualidade de vida. Paralisia cerebral. Cuidadores. Classe social.

\section{Abstract}

Introduction: Children with Cerebral Palsy (CP) have disabilities and limitations that generate dependence in relation to their caregivers. Objective: Assess whether the quality of life of caregivers of children with $C P$ is related to motor impairment, economic class and education of the caregiver. Materials and methods: We interviewed 45 informal caregivers of children with CP using the WHOQOL-bref and economic classification of Associação Brasileira de Empresas de Pesquisas (Abep). Children were classified using the Gross Motor Function Classification System. We conducted tests of association and correlation of variables with domains of the WHOQOL-bref, using independent t test and Spearman correlation coefficient. Results: A positive correlation was found between the environmental domain of WHOQOL-bref and economic status (0.56) and education of the caregiver (0.389). There were significant differences between subgroups of higher and lower economic class in the environmental ( $p=0.001)$ and psychological $(p=0.031)$, and among subgroups of high and low level of education in the environmental field $(p=0.049)$. Conclusions: The quality of life of caregivers appears to be influenced by socioeconomic level and not by the level of motor function of children with CP.

Keywords: Quality of life. Cerebral palsy. Caregivers. Social class.

\section{Introdução}

A paralisia cerebral (PC) é uma desordem permanente do movimento e postura causada por lesão não progressiva no cérebro imaturo, podendo ocorrer distúrbios de sensação, cognição, entre outros (1). É uma das patologias mais prevalentes e incapacitantes da infância (2), com demandas na área da saúde, educação e inserção social (3). A gravidade varia em cada criança e a função motora grossa é classificada pelo Gross Motor Function Classification System (GMFCS) $(4,5)$.

O impacto do nascimento de uma criança com PC implica em nova realidade para a família, com possível comprometimento da aceitação e do estabelecimento do vínculo mãe-filho $(3,6)$. A dependência funcional da criança com PC gera uma sobrecarga física e emocional associada a prejuízos na qualidade de vida de seu cuidador $(6,7)$. Estudos realizados com mães de crianças com PC verificaram indisposição para a realização de atividades físicas e para relacionamento social, má percepção da saúde, falta de conhecimento a respeito da doença do filho, diminuição do humor e problemas no convívio social, familiar e profissional $(8,9)$.
A abordagem centrada na família, modelo de intervenção com famílias de crianças com necessidades especiais, é considerada uma das melhores práticas em reabilitação pediátrica (10). Essa abordagem leva os profissionais a compreenderem o papel da família na vida da criança e a importância do conhecimento dos pais sobre as habilidades e necessidades de seu filho (10). 0 objetivo de se trabalhar com a família é melhorar a qualidade de vida de todos os seus membros, inclusive a da criança. Nesse contexto, torna-se necessário que os profissionais de saúde ofertem apoio consistente e integrado a esses indivíduos.

Segundo Oliveira, Queirós e Guerra (11), cuidador formal é o profissional que assume formalmente o exercício de cuidar, recebendo salário pela atividade. Cuidador informal é um familiar ou amigo que exerce a função de cuidar sem ressarcimento da atividade (11). A qualidade de vida é influenciada, entre outros aspectos, pela saúde física, pelo estado psicológico, pelo nível de independência, pelas relações sociais e pelo meio ambiente $(12,13)$. De acordo com uma abordagem centrada na família, conhecer o impacto da PC na qualidade de vida dos cuidadores torna mais fácil o processo de reabilitação. A promoção da qualidade de vida de cuidadores informais de crianças 
com PC - papel na maioria das vezes assumido pelas próprias mães - merece uma atenção especial (14).

0 presente estudo teve como objetivos: avaliar a qualidade de vida de cuidadores de crianças e adolescentes com PC; identificar possíveis associações entre (i) qualidade de vida do cuidador e gravidade da doença da criança, (ii) qualidade de vida do cuidador e condição econômica familiar, e (iii) qualidade de vida do cuidador e nível de escolaridade. As hipóteses são: quanto maior a gravidade da doença na criança, pior a qualidade de vida do cuidador; e quanto pior a condição socioeconômica, pior a qualidade de vida do cuidador.

\section{Materiais e métodos}

Trata-se de estudo exploratório transversal. Foram convidados a participar todos os cuidadores informais de crianças com diagnóstico de PC entre 2 e 18 anos em atendimento ambulatorial nos quatro centros de reabilitação de uma cidade do interior de Minas Gerais entre julho e outubro de 2010. A amostra, de conveniência, foi constituída por 45 cuidadores informais. 0 estudo seguiu a Resolução n. 196/96 do Conselho Nacional de Saúde e foi autorizado pelo Comitê de Ética em Pesquisa da Universidade Federal dos Vales do Jequitinhonha e Mucuri sob o parecer n. 071/10. Todos os sujeitos que consentiram em participar assinaram o Termo de Consentimento Livre e Esclarecido.

Foram coletados dados pessoais dos cuidadores como: idade, profissão, grau de escolaridade e número de filhos. Todos os sujeitos responderam a dois questionários padronizados: Questionário de Qualidade de Vida WHOQOL-bref e do critério de classificação econômica da Associação Brasileira de Empresas de Pesquisa (Abep). As crianças foram avaliadas para classificação da função motora grossa por meio do GMFCS. Em razão da dificuldade de leitura apresentada por alguns participantes, todos os questionários e avaliações foram realizados pelos pesquisadores, no centro de reabilitação frequentado por cada criança. Os pesquisadores foram treinados para aplicação dos instrumentos.

O WHOQOL-bref foi desenvolvido pela Organização Mundial de Saúde (OMS), traduzido e validado para o português (15). São 26 questões, duas gerais sobre qualidade de vida e 24 distribuídas em quatro domínios: físico, psicológico, relação social e meio ambiente $(15,16)$. As questões do WHOQOL seguem uma escala de respostas do tipo Likert: intensidade (nada - extremamente), capacidade (nada - completamente), frequência (nunca - sempre) e avaliação (muito insatisfeito - muito satisfeito; muito ruim muito bom). 0 resultado é transformado em valores quantitativos contínuos, podendo variar de 0 a $100 \%$ em cada um dos domínios, sendo que, quanto mais próximo de $100 \%$ melhor é a qualidade de vida do indivíduo $(15,16)$.

O critério de classificação econômica da Abep classifica a população em classes e subclasses econômicas a partir do número de bens duráveis que a família possui e o nível de escolaridade do chefe da família (17). São oito subclasses; a A1 refere-se à melhor classe, com pontuação entre 42 e 46, e a classe $\mathrm{E}$ refere-se à de menor poder de compra, cuja pontuação varia de 0 a 7 (17).

A classificação do nível de escolaridade informado pelo cuidador seguiu o critério da Abep. Para as análises, foi atribuída a seguinte pontuação: zero para analfabetos e cuidadores que estudaram até a terceira série do Ensino Fundamental; 1 ponto para aqueles que cursaram até a quarta série do Ensino Fundamental; 2 pontos para os que cursaram o Ensino Fundamental; 4 pontos para os que cursaram o Ensino Médio; e 8 pontos para os que cursaram o Ensino Superior completo.

O GMFCS classifica a função motora grossa de crianças com PC em cinco níveis baseados no movimento autoiniciado com ênfase na posição sentada, nas transferências e na marcha. 0 nível I refere-se à melhor função motora grossa e o nível $\mathrm{V}$ ao maior grau de limitação e dependência. Somente as crianças entre os níveis I e III são capazes de deambular sozinhas $(3,4)$.

Todos os dados foram registrados e analisados no programa SPSS versão 17.0. Para avaliar a relação entre qualidade de vida e as variáveis classe econômica, escolaridade do cuidador e GMFCS da criança, em toda a amostra, foi utilizado o Coeficiente de Correlação de Spearman, em razão da não normalidade das variáveis GMFCS, classe econômica e escolaridade. Para essa análise foram utilizados os escores brutos das variáveis. Os resultados foram interpretados da seguinte forma: valores entre 0 e 0,25 indicam correlação pequena ou nenhuma correlação; 0,25 e 0,50 , correlação regular; 0,50 e 0,75 , correlação moderada a boa; mais que 0,75 , correlação muito boa a excelente (18). 
Foi avaliada também a qualidade de vida entre os subgrupos: (i) de cuidadores de crianças com GMFCS I a III $(n=15)$ e de cuidadores de crianças com GMFCS IV e V ( $\mathrm{n}=30$ ); (ii) de cuidadores de classes econômicas A, B e C $(n=25)$ e de classes D e E $(n=20)$; e (iii) de analfabetos e pessoas com Ensino Fundamental completo e incompleto $(\mathrm{n}=$ 14) e de pessoas que cursaram Ensino Médio ou Superior $(\mathrm{n}=31)$.

A divisão foi realizada dessa forma para que fosse possível a análise estatística. Para a análise foi utilizado o Teste t para amostras independentes, devido à normalidade da variável qualidade de vida, testada pelo Teste Shapiro Wilk. 0 índice de significância escolhido para as análises foi $\alpha=0,05$.

\section{Resultados}

Dos 45 cuidadores que participaram do estudo, 42 eram mães, um era pai, uma era avó e uma era tia, com idade média de 39,4 $( \pm 9,31)$ anos. A idade média das crianças foi de 9,5 $( \pm 4,40)$ anos. A maioria dos cuidadores $(64,4 \%)$ não trabalha formalmente; $26,7 \%$ têm apenas um filho. Os valores percentuais médios de todo o grupo nos domínios do WHOQOLbref foram: físico, 69,28\%; social, 68,45\%; ambiental, 54,3\%; e psicológico, 62,51\%. As características da amostra estão descritas na Tabela 1.

0 resultado das correlações entre as variáveis GMFCS, escolaridade e classe econômica, com cada domínio do WHOQOL-bref, encontram-se na Tabela 2. O domínio ambiental apresentou correlação positiva, de magnitude moderada $(0,56)$, com a classe econômica e correlação positiva regular $(0,389)$ com a escolaridade do cuidador. Não houve correlação entre qualidade de vida do cuidador e função motora grossa da criança.

Os resultados da comparação da qualidade de vida entre os subgrupos GMFCS I, II e III e GMFCS IV e V e entre os subgrupos maior e menor escolaridade estão na Tabela 3. Não houve diferença significativa entre a qualidade de vida de cuidadores de crianças que deambulam e cuidadores de crianças que não deambulam. A qualidade de vida, no domínio ambiental, foi significativamente melhor no grupo de maior nível de escolaridade. 0 resultado da comparação da qualidade de vida entre as classes econômicas encontra-se na Tabela 4. Os cuidadores das classes econômicas mais altas (A, B e C) apresentaram, significativamente, melhores resultados nos domínios ambiental e psicológico.

Tabela 1 - Características da amostra

\begin{tabular}{|c|c|c|}
\hline Característica & $\mathbf{n}$ & $\%$ \\
\hline \multicolumn{3}{|l|}{ Sexo do cuidador } \\
\hline Feminino & 44 & 97,8 \\
\hline Masculino & 1 & 2,2 \\
\hline \multicolumn{3}{|l|}{ Profissão } \\
\hline Do lar & 29 & 64,4 \\
\hline Professor & 4 & 9,0 \\
\hline Comerciante & 2 & 4,4 \\
\hline Artesão & 1 & 2,2 \\
\hline Doméstico & 4 & 9,0 \\
\hline Escrivão & 1 & 2,2 \\
\hline Estudante & 1 & 2,2 \\
\hline Agricultor & 1 & 2,2 \\
\hline Técnico de enfermagem & 1 & 2,2 \\
\hline Desempregado & 1 & 2,2 \\
\hline \multicolumn{3}{|l|}{ Classe econômica } \\
\hline A1 e $A 2$ & 1 & 2,2 \\
\hline B1 e B2 & 7 & 15,5 \\
\hline C1 e C2 & 17 & 37,9 \\
\hline $\mathrm{D}$ & 16 & 35,6 \\
\hline$E$ & 4 & 8,8 \\
\hline \multicolumn{3}{|l|}{ Nível de escolaridade } \\
\hline Analfabeto & 2 & 4,4 \\
\hline Fundamental incompleto & 26 & 57,7 \\
\hline Fundamental completo & 1 & 2,2 \\
\hline Médio incompleto & 2 & 4,4 \\
\hline Médio completo & 8 & 17,9 \\
\hline Superior incompleto & 3 & 6,7 \\
\hline Superior completo & 3 & 6,7 \\
\hline \multicolumn{3}{|l|}{ GMFCS da criança } \\
\hline I & 5 & 11,1 \\
\hline II & 7 & 15,6 \\
\hline III & 3 & 6,7 \\
\hline IV & 11 & 24,4 \\
\hline V & 19 & 42,2 \\
\hline
\end{tabular}

Legenda: GMFCS = Gross Motor Function Classification System. Fonte: Dados da pesquisa. 
Tabela 2 - Correlação de Spearman entre os domínios do WHOQOL-bref, em toda a amostra ( $n=45)$, e as variáveis GMFCS, Classe Econômica e Escolaridade

\begin{tabular}{lccc}
\hline & GMFCS & Classe Econômica & Escolaridade \\
\hline Domínio Físico & 0,093 & 0,170 & 0,168 \\
Domínio Social & 0,113 & 0,182 & 0,088 \\
Domínio Ambiental & 0,213 & $0,560^{*}$ & $0,389^{*}$ \\
Domínio Psicológico & 0,039 & 0,282 & 0,262 \\
\hline
\end{tabular}

Legenda: GMFCS = Gross Motor Function Classification System; ${ }^{*}=$ Teste de Spearman significativo para $p \leq 0,05$.

Fonte: Dados da pesquisa.

Tabela 3 - Comparações entre as médias dos domínios do WHOQOL-bref nos subgrupos da variável GMFCS da criança e Nível de Escolaridade do cuidador utilizando-se o Teste t para amostras independentes

\begin{tabular}{lccccccc}
\hline & \multicolumn{3}{c}{ GMFCS } & & \multicolumn{3}{c}{ Nível de escolaridade } \\
\cline { 2 - 3 } & I, II e III & IV e V & $\mathbf{p}$ & & Médio/Superior & Analfabeto/Fundamental & p \\
\hline Sujeitos (n) & 15 & 30 & & & 14 & 31 & \\
Domínio Físico & $69,90 \pm 18,95$ & $68,97 \pm 13,66$ & 0,850 & & $72,1 \pm 9,60$ & $68,00 \pm 17,41$ & 0,416 \\
Domínio Social & $66,04 \pm 17,39$ & $69,65 \pm 14,93$ & 0,473 & & $68,98 \pm 12,42$ & $68,20 \pm 17,14$ & 0,879 \\
Domínio Ambiental & $51,37 \pm 16,70$ & $55,77 \pm 16,67$ & 0,408 & & $61,53 \pm 12,84$ & $51,04 \pm 17,28$ & $0,049^{*}$ \\
Domínio Psicológico & $64,9 \pm 13,18$ & $61,31 \pm 15,78$ & 0,452 & & $65,41 \pm 14,9$ & $61,19 \pm 15,30$ & 0,385 \\
\hline
\end{tabular}

Legenda: GMFCS $=$ Gross Motor Function Classification System; * $=$ Teste t significativo para $p \leq 0,05$.

Fonte: Dados da pesquisa.

Tabela 4 - Comparação entre as médias dos domínios do WHOQOL-bref nos subgrupos da variável Classe Econômica utilizando-se 0 Teste t para amostras independentes

\begin{tabular}{lccc}
\hline & $\begin{array}{c}\text { Classes } \\
\text { A, B e C }\end{array}$ & $\begin{array}{c}\text { Classes } \\
\text { D e E }\end{array}$ & p \\
\hline Sujeitos (n) & 25 & 20 & 0,159 \\
Domínio Físico & $72,20 \pm 12,61$ & $65,64 \pm 13,00$ & 0,697 \\
Domínio Social & $69,28 \pm 14,18$ & $67,41 \pm 17,72$ & $0,001^{*}$ \\
Domínio Ambiental & $61,43 \pm 17,22$ & $45,39 \pm 10,71$ & $0,031^{*}$ \\
Domínio Psicológico & $66,76 \pm 15,71$ & $57,20 \pm 12,24$ & \\
\hline
\end{tabular}

Legenda: ${ }^{*}=$ Teste t significativo para $p \leq 0,05$.

Fonte: Dados da pesquisa.

\section{Discussão}

O presente estudo explorou três fatores que poderiam interferir na qualidade de vida de cuidadores de crianças com PC. Tanto a análise de correlação quanto a análise comparativa entre grupos confirmou a hipótese que uma pior qualidade de vida está relacionada a uma pior condição socioeconômica, mas não confirmou a hipótese com relação ao comprometimento motor grosso da criança.

O domínio ambiental foi o que apresentou menor valor médio em toda a amostra. Foi o único domínio 
que apresentou, nas duas análises, resultados significativos com relação às variáveis escolaridade $\mathrm{e}$ classe econômica. Esse domínio, no WHOQOL-bref, diz respeito ao acesso a saúde, transporte, lazer, recursos financeiros e segurança (16) - questões diretamente relacionadas à condição financeira familiar. De fato, segundo a literatura, uma menor condição socioeconômica está relacionada a uma menor qualidade de vida $(15,19,20)$.

Em estudo com cuidadores de crianças autistas, Barbosa e Fernandes (21), utilizando o WHOQOLbref, também identificaram diferenças significativas no domínio ambiental, quando relacionado com escolaridade e classe econômica. Os fatores referentes ao domínio ambiental, como dificuldade de acesso a serviços de necessidade básica, contribuem para o aumento do nível de estresse de cuidadores (21). Nossos resultados corroboram com os resultados de Barbosa e Fernandes e apontam para a necessidade de o governo e os profissionais da área oferecerem acesso a serviços de saúde, transporte, recreação e lazer, tanto das crianças quanto de seus cuidadores.

Segundo a literatura, mães de crianças com necessidades especiais tendem a se isolar socialmente, reduzindo o apoio que poderiam receber da sociedade $(20,22)$. Neste estudo não foi encontrada relação significativa entre o domínio social, que analisa o apoio que o cuidador recebe de familiares e amigos, com nenhuma das variáveis estudadas. Oliveira et al. (23) observaram que, apesar de apresentarem qualidade de vida insatisfatória, mães de crianças com PC encontram apoio e conforto na religiosidade.

Mães de crianças com PC apresentam maior prevalência de depressão que mães de crianças sem deficiências (24). Neste estudo o domínio psicológico apresentou relação siginificativa apenas com a classe econômica. Sajedi et al. (24) também não encontraram diferença entre o grau de distúrbio psicológico nas mães e o GMFCS da criança. Manuel et al. (25) encontraram sintomas de depressão em $30 \%$ das mães estudadas, porém estes não estavam relacionados com o comprometimento motor de seus filhos. Núñez (26) observou que conflitos familiares não surgem em resultado direto da deficiência, mas em função da possibilidade de a família se adaptar ou não a situações de estresse.

A hipótese de que um maior comprometimento da função motora grossa da criança acarretaria pior qualidade de vida do cuidador, especialmente no domínio físico, não foi confirmada neste estudo. Camargos et al. (27) encontraram uma maior sobrecarga em cuidadores de crianças com PC das classes econômicas menos favorecidas; no entanto, foi encontrada maior sobrecarga entre cuidadores de crianças com menor comprometimento motor. Apesar de o GMFCS avaliar a capacidade de locomoção, a independência funcional da criança depende ainda de outros fatores como capacidade manual e cognitiva. Este pode ser o motivo de vários estudos não encontrarem relação entre a qualidade de vida do cuidador e o GMFCS da criança $(24,25,27,28)$.

Apesar de o domínio físico não ter sido significativamente relacionado às variáveis estudadas, neste estudo, todos os cuidadores relataram apresentar dor física e $9 \%$ afirmaram que a dor os impede bastante ou extremamente de desempenharem as atividades do dia a dia. Outros estudos demonstram que cuidadores de crianças com necessidades especiais apresentam menor desempenho físico, dores no corpo, diminuição da vitalidade, estresse e pouco tempo para a prática de atividades físicas $(9,23,29$, 30). A saúde física dos cuidadores de crianças com PC deve ser alvo de cuidados dos profissionais de Saúde, independente da condição socioeconômica ou gravidade da patologia na criança.

\section{Conclusão}

Neste estudo a qualidade de vida de cuidadores de crianças e adolescentes com PC demonstrou ser mais influenciada pela condição socioeconômica e escolaridade que pela gravidade da função motora grossa da criança com PC. Outros estudos devem ser realizados com amostras maiores, de forma a permitir a formação de subgrupos para todas as classes econômicas, todos os níveis de escolaridade e de GMFCS. Sugerem-se ainda estudos que levem em consideração outros aspectos das deficiências apresentadas pela criança, como independência nas atividades de vida diária, para melhor entendimento dos fatores que podem interferir na qualidade de vida de seus cuidadores.

\section{Referências}

1. Rosenbaum P, Paneth N, Leviton A, Goldstein M, Bax MA. A report: the definition and classification of cerebral palsy. Dev Med Child Neurol. 2007;49(S109):8-14. 
2. Cans C. Surveillance of cerebral palsy in Europe: a collaboration of cerebral palsy surveys and registers. Dev Med Child Neurol. 2000;42:816-24. doi:10.1111/j.1469-8749.2000.tb00695.x.

3. White-Koning M, Grangjean H, Colver A, Arnaud C. Parent and professional reports of the quality of life of children with cerebral palsy and associated intellectual impairment. Dev Med Child Neurol. 2008;50(8):61824. doi:10.1111/j.1469-8749.2008.03026.x.

4. Palisano RJ, Rosenbaum P, Bartlett D, Livingston MH. Content validity of the expanded and revised Gross Motor Function Classification System. Dev Med Child Neurol. 2008;50:744-50. doi:10.1111/ j.1469-8749.2008.03089.x.

5. Hiratuka E, Matsukura TS, Pfeifer LI. Adaptação transcultural para o Brasil do sistema de classificação da função motora grossa. Rev Bras Fisioter. 2012;14(6):53744. doi:10.1590/S1413-35552010000600013.

6. Petean EBL, Murata MF. Paralisia cerebral: conhecimento das mães sobre o diagnóstico e o impacto deste na dinâmica familiar. Paidéia. 2000;10(19):40-46. doi:10.1590/S0103-863X2000000200006.

7. Lopes GB, Kato LS, Corrêa PRC. Os pais das crianças com deficiência: reflexões acerca da orientação em reabilitação motora. Psicol Teor Prat. 2002;4(2):67-72.

8. Mugno D, Ruta L, D’Arrigo V, Mazzone L. Impairment of quality of life in parents of children and adolescents with pervasive developmental disorder. Health Qual Life Outcomes. 2007;5:22-30. doi:10.1186/ 1477-7525-5-22.

9. Ones K, Yilmaz E, Cetinkaya B, Caglar N. Assessment of the quality of life of mothers of children with cerebral palsy (primary caregivers). Neurorehabil Neural Repair. 2005;19(3):232-7. doi:10.1177/ 1545968305278857.

10. King S, Teplicky R, King G, Rosenbaum P. Family-centered service for children with cerebral palsy and their families: a review of the literature. Semin Pediatr Neurol. 2004;11(1):78-86. doi:10.1016/j. spen.2004.01.009.

11. Oliveira MA, Queirós C, Guerra MP. O conceito de cuidador analisado numa perspectiva autopoiética: do caos à autopoiése. Psic, Saúde \& Doenças. 2007; 8(2):181-96.
12. Seidl EMF, Zannon CMLC. Qualidade de vida e saúde: aspectos conceituais e metodológicos. Cad Saúde Pública. 2004;20(2):580-88. doi:10.1590/ S0102-311X2004000200027.

13. The WHOQOL Group. The World Health Organization quality of life assessment (WHOQOL): position paper from the World Health Organization. Soc Sci Med. 1995;41:1403-10. doi:10.1016/ 0277-9536(95)00112-K.

14. Carvalho JTM, Rodrigues NM, Silva LVC, Oliveira DA. Qualidade de vida das mães de crianças e adolescentes com paralisia cerebral. Fisioter Mov. 2010;23(3):38997. doi:10.1590/S0103-51502010000300006.

15. Fleck MPA, Louzada S, Xavier M, Chachamovich E, Vieira G, Santos L, et al. Aplicação da versão em português do instrumento abreviado de avaliação da qualidade de vida "WHOQOL-bref". Rev Saúde Públ. 2000;34(2):178-83. doi:10.1590/ S0034-89102000000200012.

16. Organização Mundial de Saúde - OMS. Divisão de Saúde Mental. Grupo WHOQOL. Versão em português dos instrumentos de avaliação de qualidade de vida (WHOQOL) 1998; [acesso 15 jun. 2010]. Disponível em: www.ufrgs.br/psiq/whoqol-100.html.

17. Associação Brasileira de Empresas de Pesquisa. Critério de Classificação econômica Brasil; [acesso 22 jun. 2010]. Disponível em: www.abep.org/novo/Content. aspx?ContentID=301.

18. Dawson B, Trapp RG. Basic \& Clinical Biostatistics. 3rd. ed. Lange Medical Books/McGraw-Hill: Medical Publishing Division; 2001.

19. Oliveira SC. Conceitos e generalizações sobre qualidade de vida. Rev Cent Ciênc Saúde. 2002;15(1):1-7.

20. Monteiro M, Matos AP, Coelho R. Adaptação psicológica de mães cujos filhos apresentam paralisia cerebral - resultados de um estudo. Rev Portuguesa de Psicossomática. 2004;6(1):115-30.

21. Barbosa MRP, Fernandes FDM. Qualidade de vida dos cuidadores de crianças com transtorno do espectro autístico. Rev Soc Bras Fonoaudiol. 2009;14(3):482-6. doi:10.1590/S1516-80342009000400009.

22. Sousa SCB, Pires AAP. Comportamento materno em situação de risco: mães de crianças com paralisia cerebral. Psic, Saúde \& Doenças. 2003;4(1):111-30. 
23. Oliveira MFS, Silva MBM, Frota MA, Pinto JMS, Frota LMCP, Sá FE. Qualidade de vida do cuidador de crianças com paralisia cerebral. Rev Bra Promoção a Saúde. 2008;21(4):275-80. doi:10.5020/18061230.2008. p275.

24. Sajedi F, Alizad V, Malekkhosravi G, Karimlou M, Vameghi R. depression in mothers of children with cerebral palsy and its relation to severity and type of cerebral palsy. Acta Med Iranica. 2010;48(4):250-4. PMid:21279939.

25. Manuel J, Naughton MJ, Balkrishnan R, Smith BP, Koman A. Stress and adaptation in mothers of children with cerebral palsy. J Pediatr Psychol. 2003;28(3):197201. doi:10.1093/jpepsy/jsg007.

26. Núñez B. La familia con un hijo con discapacidad: sus conflictos vinculares. Arch Argent Pediatr. 2003; 101(2):133-42.

27. Camargos ACR, Lacerda TTB, Viana SO, Pinto LRA, Fonseca MLS. Avaliação da sobrecarga do cuidador de crianças com Paralisia Cerebral através da escala Burden Interview. Rev Bras Saúde Matern Infant. 2009;9(1):3137. doi:10.1590/S1519- 38292009000100004.
28. Tuna H, Unalan H, Tuna F, Kokino S. Quality of life of primary caregivers of children with cerebral palsy: a controlled study with short from-36 questionnaire. Dev Med Child Neurol. 2004;46:646-48. doi:10.1017/ S0012162204221077.

29. Eker L, Tuzun EH. An evaluation of quality of life of mothers of children with cerebral palsy. Disabil Rehabil. 2004;26(23):1354-9. doi:10.1080/ 09638280400000187.

30. Almeida T, Sampaio FM. Stress e suporte social em familiares de pessoas com paralisia cerebral. Psic, Saúde \& Doenças. 2007;8(1):145-51.

Recebido: $17 / 08 / 2012$

Received: 08/17/2012

Aprovado: 07/01/2013

Approved: 01/07/2013 